\title{
Electron Tomographic Studies of Mitochondrial Crista Topology: "Swirl" Mitochondria of Drosophila Flight Muscle
}

\author{
C.A. Mannella*, K.F. Buttle*, D.W. Walker**, S. Benzer** \\ * Resource for Visualization of Biological Complexity, Wadsworth Center, Empire State Plaza, \\ Box 509, Albany, NY 12201-0509 \\ ** Division of Biology, 156-29, California Institute of Technology, Pasadena, CA 91125
}

There is no better example of the impact of electron tomography on our understanding of cell ultrastructure than the mitochondrion. Mitochondrial cristae were, until recently, represented as random infoldings of the inner membrane, the site of the machinery of oxidative phosphorylation. Electron tomography has revealed, instead, that the cristae define micro-compartments interconnected by narrow tubular regions to each other and to the intermembrane space [1]. There is evidence that the topology of the inner membrane influences mitochondrial functions by its effects on internal diffusion of metabolites and soluble proteins $[2,3]$. It has been proposed that the topology of this membrane is a regulated property of mitochondria, under the control of proteins and lipids that affect membrane curvature and fission/fusion [4].

There are numerous examples of aberrant mitochondrial inner membrane topologies that likely represent dysfunction of the usual control mechanisms. An example is the distinctive "swirls" of inner membranes observed in flight-muscle mitochondria of Drosophila melanogaster under conditions of oxygen stress or under normal oxygen tension in a hyperswirl mutant [5]. The swirl regions are composed of inner membranes that have lost their usual tight, orderly packing, and are deficient in cytochrome oxidase activity [5]. Tomographic analysis is being used to unravel the organization of the membranes within the swirls. In the mitochondrion reconstructed in Fig.1, the swirl is composed of five parallel rows of membranes whose outlines (in slices from the tomogram) vary from circular to elongated (Fig.1B-D). 3D views of the membrane surfaces indicate that the membranes are organized as a bundle of undulating 20-30-nm-diameter tubes that extend hundreds of nm along the length of the swirl (Fig.1E,F). The region of denser membrane packing in the middle of the swirl (arrows in Fig.1C) may represent an intermediate stage in formation of the tubes. The membranes in this region are flattened tubes with interior dimensions ca. 15-nm x 70-nm which terminate on either end in dilated compartments contiguous with the tubular bundle. Interestingly, the unusual morphology of these mitochondrial inner membranes bears some resemblance to the paracrystalline membrane morphology observed in mitochondria of starved amoeba, associated with elevated oxygen radical generation [6]. This could suggest a generalized mitochondrial structural response to oxidative stress, with possible relevance to aging and neurodegenerative diseases.

\section{References}

[1] T. Frey and C.A. Mannella, Trends Biochem. Sci. 25 (2000) 319.

[2] Mannella et al., IUBMB Life 52 (2001) 93.

[3] Scorrano et al., Devel. Cell 138 (2002) 63.

[4] Mannella, Biochim. Biophys. Acta 1763 (2006) 542.

[5] D.W. Walker and S. Benzer, Proc. Natl. Acad. USA 101 (2004) 10290. 
[6] Deng et al., Protoplasma 219 (2002) 160.

[7] Supported by NCRR/NIH grant RR01219 and the Ellison Medical Foundation.
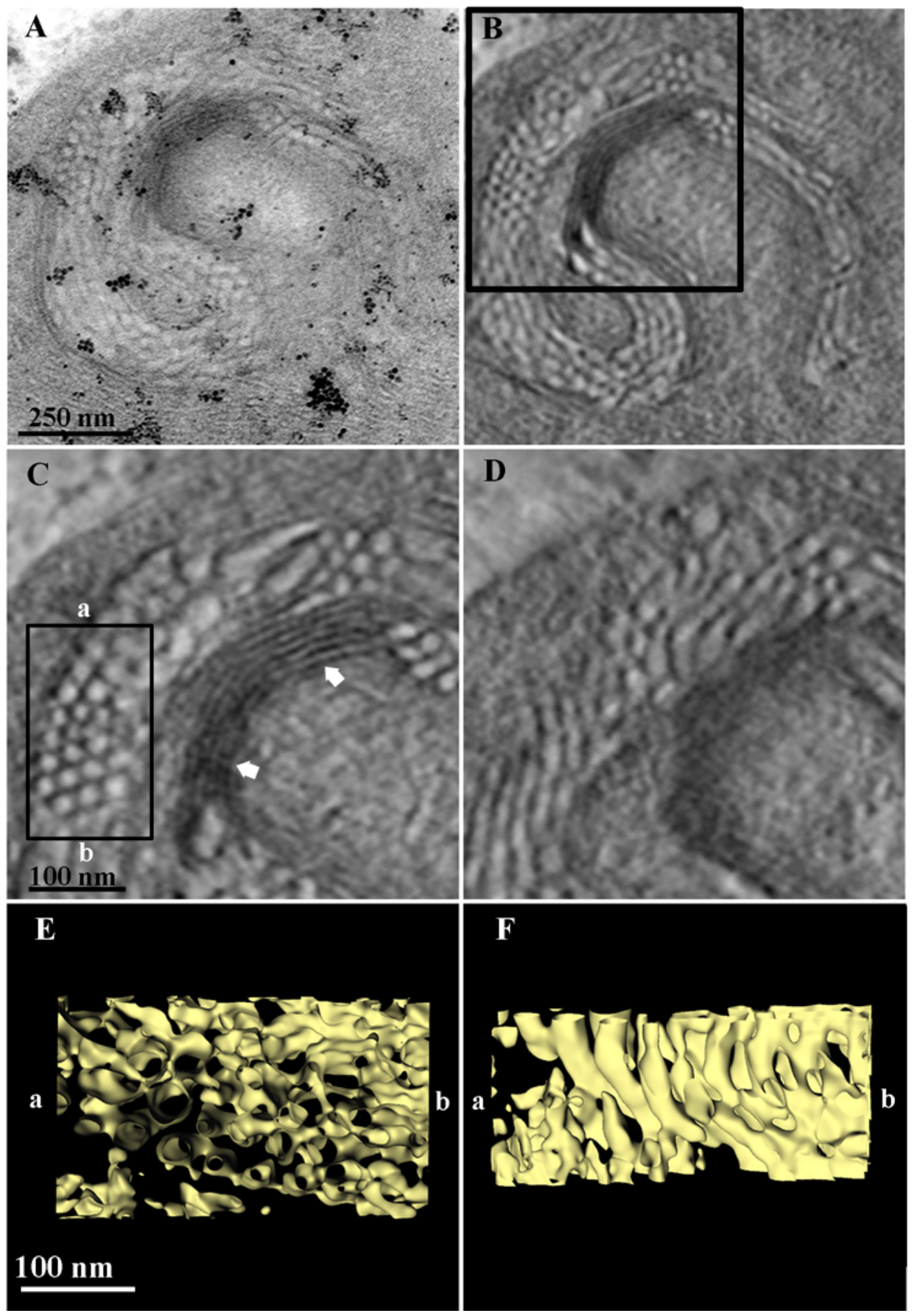

Fig.1. Tomographic analysis of a "swirl" mitochondrion. A. Projection through a 185-nm-thick plastic section [5]. B. Single slice (1.3-nm-thick) taken from the center of the tomogram. C,D. Slices of the subregion boxed in B, taken $56 \mathrm{~nm}$ and $153 \mathrm{~nm}$, respectively, from the top of the tomogram. E,F. Top and side views of an isodensity surface model illustrating the curved tubular topology of the inner membranes in the region indicated by the box in $\mathrm{C}$. 